\title{
Injectiveness and Discontinuity of Multiplicative Convex Functions
}

\author{
Pablo Jiménez-Rodríguez ${ }^{1,+}$, María E. Martínez-Gómez ${ }^{2, \dagger}$, Gustavo A. Muñoz-Fernández ${ }^{3,+}$ \\ and Juan B. Seoane-Sepúlveda ${ }^{3, *,+}$ (D) \\ 1 Departamento de Matemática Aplicada, Campus Duques de Soria, Universidad de Valladolid, \\ 42004 Soria, Spain; pablo.jimenez.rodriguez@uva.es \\ 2 Departamento de Análisis Matemático y Matemática Aplicada, Facultad de Ciencias Matemáticas, \\ Universidad Complutense de Madrid, Plaza de Ciencias 3, 28040 Madrid, Spain; mariae24@ucm.es \\ 3 Departamento de Análisis Matemático y Matemática Aplicada, Facultad de Ciencias Matemáticas, \\ Instituto de Matemática Interdisciplinar (IMI), Plaza de Ciencias 3, Universidad Complutense de Madrid, \\ 28040 Madrid, Spain; gustavo_fernandez@mat.ucm.es \\ * Correspondence: jseoane@ucm.es \\ + All authors contributed equally to this work.
}

check for

updates

Citation: Jiménez-Rodríguez, P.;

Martínez-Gómez, M.E.;

Muñoz-Fernández, G.A.;

Seoane-Sepúlveda, J.B. Injectiveness and Discontinuity of Multiplicative

Convex Functions. Mathematics 2021, 9, 1035. https://doi.org/10.3390/ math9091035

Received: 27 March 2021

Accepted: 28 April 2021

Published: 3 May 2021

Publisher's Note: MDPI stays neutral with regard to jurisdictional claims in published maps and institutional affiliations.

Copyright: (C) 2021 by the authors. Licensee MDPI, Basel, Switzerland. This article is an open access article distributed under the terms and conditions of the Creative Commons Attribution (CC BY) license (https:/ / creativecommons.org/licenses/by/ $4.0 /)$.

\begin{abstract}
In the present work we study the set of multiplicative convex functions. In particular, we focus on the properties of injectiveness and discontinuity. We will show that a non constant multiplicative convex function is at most 2-injective, and construct multiplicative convex functions which are discontinuous at infinitely many points.
\end{abstract}

Keywords: convexity; convex function; multiplicative convex

\section{Introduction and Background Research on the Set of Multiplicative Convex Functions}

The class of multiplicative convex functions was introduced as a way of extending the property of classical convexity from the arithmetic mean to the geometric mean. With this idea, Niculescu proposed that a multiplicative convex function would verify the relation

$$
f\left(x^{\lambda} y^{1-\lambda}\right) \leq f(x)^{\lambda} f(y)^{1-\lambda}
$$

for every choice of $x, y>0$ and $0<\lambda<1$ ([1]). On the other hand, in [2] the authors proposed a different way to define multiplicative convexity were the aim was to upgrade the classical definition from addition to multiplication. In this direction, the following definition was introduced:

Definition $1([3])$. A function $f:(0, \infty) \rightarrow[0, \infty)$ is said to be multiplicative convex (or $f$ is mc) if, for every $\mu>0$ and $x, y \geq 0$ we have

$$
f\left(x^{\mu} y^{1 / \mu}\right) \leq f(x)^{\mu} f(y)^{1 / \mu} .
$$

If, besides, $f(1)=1$ then we will say that $f$ is multiplicative convex $\mathbf{1}$ (or mc1, for short).

We will denote

$$
\begin{aligned}
\mathcal{M C} & =\{f:(0, \infty) \rightarrow[0, \infty): \text { fis } m c\}, \\
\mathcal{M C} 1 & =\{f:(0, \infty) \rightarrow[0, \infty): \text { fis } m c 1\}
\end{aligned}
$$

In [2] it was shown that the distinction between the sets $\mathcal{M C}$ and $\mathcal{M C} 1$ (namely, $f(1)=1$ ) was crucial. In fact, the condition $f(1)=1$ suffices to completely describe the set $\mathcal{M C} 1$ :

Theorem 1 ([2]). Let $f:(0, \infty) \rightarrow[0, \infty)$. Then, $f$ is an mc1-function if and only if it can be written in the form 


$$
f(x)= \begin{cases}b^{\log _{a}(x)} & \text { if } 0<x \leq 1, \\ b^{\prime \log _{a^{\prime}}(x)} & \text { if } x>1,\end{cases}
$$

where $a, b, a^{\prime}$ and $b^{\prime}$ satisfy the following conditions:

1. $0<a<1$ and $a^{\prime}>1$.

2. If $b<1$, then $\log _{b}\left(b^{\prime}\right) \leq \log _{a}\left(a^{\prime}\right)<0$ (which, in particular, implies $b^{\prime}>1$ ).

3. If $b>1$, then $\log _{b}\left(b^{\prime}\right) \geq \log _{a}\left(a^{\prime}\right)$.

In particular, Theorem 1 implies that an $m c 1$-function can show one (and only one) of the following behaviors: it is either monotone (increasing or decreasing) or it decreases over the interval $(0,1)$ and increases over the interval $(1, \infty)$. Functions that behave like the latter ones (decrease over $(0, b)$ and increase over $(b, \infty)$ for certain number $b>0$ ) will be called decreasing-increasing functions.

On the other hand, requiring $f(1)>1$ (for an $m c$-function cannot have $f(1)<1$ ) implied a huge difference with respect to the previous situation, where everything was under control: for one thing (see [3]), $\mathcal{M C}$ is closed under addition, product, multiplication by a scalar no smaller than 1 and composition (if the first function acting in the composition is not decreasing), but it was also proved the existence of discontinuous $m c$-functions:

Theorem 2 ([3]). Let $\alpha>1, \alpha<\beta \leq \alpha^{2}$ and $f$ be an mc1-function. Define the function

$$
g(x)= \begin{cases}\alpha f(x) & \text { if } 0<x \leq 1 \\ \beta f(x) & \text { if } x>1\end{cases}
$$

Then $g$ is a discontinuous mc-function.

The following theorem can be used to prove the existence of an $m c$-function which is discontinuous at any given point. We include the constructive proof for the sake of completeness.

Proposition 1 ([3]). Let $x_{0}>0$. Then, there exists an mc-function that is discontinuous at $x_{0}$

Proof. Assume first $x_{0}<1$ and let $f$ be an $m c 1$-function and $g$ be an increasing discontinuous function from Theorem 2 . Then,

$$
h_{x_{0}}(x)=g\left(\frac{1}{f\left(x_{0}\right)} f(x)\right)
$$

is an $m c$-function which is discontinuous at $x_{0}$.

If now $x_{0}>1$, then we may just define

$$
h^{\left(x_{0}\right)}(x)=h_{\frac{1}{x_{0}}}\left(\frac{1}{x}\right) .
$$

Given the aim of this paper, we shall introduce some notation to denote the set of points of discontinuity of functions.

Definition 2. Let $f: \mathbb{R} \rightarrow \mathbb{R}$ be a function. We shall denote

$$
\mathfrak{D}(f)=\{x \in \mathbb{R}: f \text { is discontinuous on } x\} .
$$


This set has some regular algebraic properties. For example, what follows is a standard calculus exercise:

Proposition 2. Let $f$ and $g$ be two functions so that $\mathfrak{D}(f) \cap \mathfrak{D}(g)=\varnothing$. Then,

$$
\mathfrak{D}(f+g)=\mathfrak{D}(f g)=\mathfrak{D}(f) \cup \mathfrak{D}(g) .
$$

In this paper we have two main objectives: the first one is to complete a description of the set of general $m c$-functions in a similar way as how the set of $m c 1$-functions was described in [2] (where, before succeeding in giving a complete characterization of the set, the authors proved that $m c 1$-functions were continuous and either monotone or non increasing-non decreasing). The results in Section 3 will lead to the conclusions that a general $m c$-function is of one of the mentioned two behaviors (monotone or decreasingincreasing), which in particular implies that the set $\mathcal{M C}$ is of cardinality $\mathfrak{c}$ and that an $m c$-function is continuous everywhere except from possibly a countable set.

The second aim is to provide examples of $m c$-functions which are discontinuous at infinitely many points. The main result in Section 4 is held by some propositions and lemmas encompassed in Number Theory.

We will complete this paper by analyzing the set of discontinuous $m c$-functions from an algebraic point of view. Section 5 follows the line of action shown in [3] and focuses on the existence of certain algebraic structures whose non-zero elements fulfill some properties (see, e.g., [4-8]).

\section{Other Fundamental Concepts and Classical Results}

The following two theorems will be of great importance for the goals of this paper.

Theorem 3 ([9], Darboux-Froda's Theorem). The set of points of discontinuity of a monotone function is at most countable.

Theorem 4 ([10]). The cardinality of the set of monotone functions is $\mathfrak{c}$.

Besides this, we will also make use of Steiner's problem, which we include bellow:

Lemma 1 ([11]). The maximum of the function $f(x)=x^{1 / x}$ is attained at $x=e$. In fact, the function $f$ is increasing on $(0, e)$ and decreasing on $(e, \infty)$.

With respect to the algebraic properties of the set $\mathcal{M C}$, we remark that in general we cannot ensure that, given an $m c$-function $f, \alpha f$ is also an $m c$-function for $\alpha \geq 0$ but for $\alpha \geq 1$. Because of this, we have to consider the following definition.

Definition 3 ([3]). Let $X$ be a vector space and $M \subseteq X$. We say that $M$ is an infinite dimensional truncated cone if $M$ fulfills the following properties:

1. $M$ is closed under addition.

2. $\quad M$ is closed under multiplication by scalars greater than or equal to 1 .

3. $M$ contains a set of infinite cardinality of linearly independent elements.

The maximal possible cardinality of a set as in property (3) is the dimension of the truncated cone. If, furthermore, we can define a multiplication on X (satisfying the usual properties on itself and with respect to addition) and $M$ is closed under multiplication, then we say that $M$ is an algebraic truncated cone. In that case,

1. the linear dimension of the truncated cone will be the maximal possible cardinality so that there exists a subset of such cardinality and consisting on linearly independent elements.

2. The algebraic dimension will be the maximal possible cardinality so that there exists a subset of such cardinality and consisting on algebraic independent elements (that is, so that the only polynomial vanishing on them is the null polynomial). 
Trivially, we obtain that the algebraic dimension is not greater than the linear dimension.

\section{Study of the Injectiveness of an $m c$-Function}

In order to conclude that an $m c$-function $f$ is either monotone or decreasing-increasing, we will first show that we have that $f_{\mid f^{-1}(0,1)}$ is injective and that $f_{\mid f^{-1}[1, \infty)}$ is at most 2-injective.

Theorem 5. Let $f$ be an mc-function and define $A=f^{-1}(0,1)$. Then $f_{\mid A}$ is injective.

Proof. Assume otherwise, that is, we can find $0<\theta_{1}<\theta_{2}$ so that $f\left(\theta_{1}\right)=f\left(\theta_{2}\right)=c<1$. Assume furthermore that $1<\theta_{1}, \theta_{2}$. We claim that we must have $\theta_{2}<\theta_{1}^{4}$. Indeed, if $\theta_{2} \geq \theta_{1}^{4}$, then

$$
\begin{aligned}
& 1=\log _{\theta_{2}}\left(\theta_{2}\right) \geq 4 \log _{\theta_{2}}\left(\theta_{1}\right) \quad \text { so } \\
& 0 \leq 1-4 \log _{\theta_{2}}\left(\theta_{1}\right)
\end{aligned}
$$

and therefore we can define

$$
\mu=\frac{1+\sqrt{1-4 \log _{\theta_{2}}\left(\theta_{1}\right)}}{2}>0,
$$

which is a solution to $\theta_{1}^{1 / \mu} \theta_{2}^{\mu}=\theta_{2}$. This allows us to conlcude

$$
f\left(\theta_{2}\right)=f\left(\theta_{1}^{1 / \mu} \theta_{2}^{\mu}\right) \leq f\left(\theta_{1}\right)^{1 / \mu} f\left(\theta_{2}\right)^{\mu}=f\left(\theta_{2}\right)^{\mu+1 / \mu},
$$

finding a contradiction to the condition $f\left(\theta_{2}\right)<1$ and proving our claim.

Define next

$$
g(x)=f\left(\frac{\theta_{1}^{4}}{\theta_{2}} x\right)
$$

Then,

$$
g\left(\frac{\theta_{1} \theta_{2}}{\theta_{1}^{4}}\right)=g\left(\frac{\theta_{2}^{2}}{\theta_{1}^{4}}\right)
$$

so by our previous claim,

$$
\begin{aligned}
\frac{\theta_{2}^{2}}{\theta_{1}^{4}} & <\left(\frac{\theta_{1} \theta_{2}}{\theta_{1}^{4}}\right)^{4}=\frac{\theta_{1}^{4} \theta_{2}^{4}}{\theta_{1}^{16}} \quad \text { from which } \\
\theta_{1}^{8} & <\theta_{2}^{2}
\end{aligned}
$$

arriving at a contradiction.

If $0<\theta_{1}<1<\theta_{2}$, then $\log _{\theta_{2}}\left(\theta_{1}\right)<0$ and therefore

$$
\mu=\frac{1+\sqrt{1-4 \log _{\theta_{2}}\left(\theta_{1}\right)}}{2}
$$

is always well-defined and positive, so we can again arrive at the contradiction $f\left(\theta_{2}\right) \leq$ $f\left(\theta_{2}\right)^{\mu+1 / \mu}$.

Any other situation may be reduced to one of the previous two via the auxiliary function $h(x)=f(1 / x)$.

Theorem 6. There is no mc-function, $f$, so that we can find $1<\theta_{1}<\theta_{2}$ such that $\max \{f(1)$, $\left.f\left(\theta_{2}\right)\right\}<f\left(\theta_{1}\right)$. 
Proof. If we choose

$$
\mu=\log _{\theta_{2}}\left(\theta_{1}\right)<1
$$

then it must be

$$
f\left(\theta_{1}\right) \leq f\left(\theta_{2}\right)^{\mu} f(1)^{1 / \mu} .
$$

Let us choose $0<a, b<1, a^{\prime}, b^{\prime}>1$, and $\alpha>1$ so that

$$
\begin{aligned}
\log _{b}\left(b^{\prime}\right) & =\log _{a}\left(a^{\prime}\right), \\
\alpha b^{\prime \log _{a^{\prime}}\left(\theta_{1}\right)} f\left(\theta_{1}\right) & >f(1), \\
b^{\prime \log _{a^{\prime}}\left(\frac{\theta_{1}}{\theta_{2}}\right)} f\left(\theta_{1}\right) & >f\left(\theta_{2}\right), \\
\alpha^{2-(2 \mu+1 / \mu)} & >\frac{f\left(\theta_{2}\right)^{\mu} f(1)^{1 / \mu}}{f\left(\theta_{1}\right)} .
\end{aligned}
$$

Then, if we define the function

$$
g(x)= \begin{cases}\alpha b^{\log _{a}(x)} & \text { if } 0<x \leq 1, \\ \alpha^{2} b^{\prime \log a^{\prime}(x)} & \text { if } x>1,\end{cases}
$$

we can apply Theorems 1 and 2 to conclude that $g$ is a (discontinuous) $m c$-function. Hence, $h(x)=f(x) g(x)$ would also be an $m c$-function. Now, we can deduce the following chain of equivalent inequalities:

$$
\begin{aligned}
& \alpha^{2-\left(2 \log _{\theta_{2}}\left(\theta_{1}\right)+1 / \log _{\theta_{2}}\left(\theta_{1}\right)\right)}>\frac{f\left(\theta_{2}\right)^{\log _{\theta_{2}}\left(\theta_{1}\right)} f(1)^{1 / \log _{\theta_{2}}\left(\theta_{1}\right)}}{f\left(\theta_{1}\right)}, \\
& \alpha^{2} f\left(\theta_{1}\right)>\left[\alpha^{2} f\left(\theta_{2}\right)\right]^{\log _{\theta_{2}}\left(\theta_{1}\right)}[\alpha f(1)]^{1 / \log _{\theta_{2}}\left(\theta_{1}\right)}, \\
& \alpha^{2} b^{\log _{a}\left(\theta_{1}\right)} f\left(\theta_{1}\right)>\left[\alpha^{2} f\left(\theta_{2}\right)\right]^{\log _{\theta_{2}}\left(\theta_{1}\right)} b^{\log _{a}\left(\theta_{1}\right)}[\alpha f(1)]^{1 / \log _{\theta_{2}}\left(\theta_{1}\right)}, \\
& \alpha^{2} b^{\log _{a}\left(a^{\prime}\right) \log _{a^{\prime}}\left(\theta_{1}\right)} f\left(\theta_{1}\right)>\left[\alpha^{2} f\left(\theta_{2}\right)\right]^{\log _{\theta_{2}}\left(\theta_{1}\right)} b^{\log _{a}\left(a^{\prime}\right) \log _{a^{\prime}}\left(\theta_{2}\right) \log _{\theta_{2}}\left(\theta_{1}\right)} \\
& \cdot[\alpha f(1)]^{1 / \log _{\theta_{2}}\left(\theta_{1}\right)}, \\
& \alpha^{2} b^{\log _{b}\left(b^{\prime}\right) \log _{a^{\prime}}\left(\theta_{1}\right)} f\left(\theta_{1}\right)>\left[\alpha^{2} b^{\log _{b}\left(b^{\prime}\right) \log _{a^{\prime}}\left(\theta_{2}\right)} f\left(\theta_{2}\right)\right]^{\log _{\theta_{2}}\left(\theta_{1}\right)} \\
& \cdot[\alpha f(1)]^{1 / \log _{\theta_{2}}\left(\theta_{1}\right)} \text {, } \\
& \alpha^{2} b^{\prime \log _{a^{\prime}}\left(\theta_{1}\right)} f\left(\theta_{1}\right)>\left[\alpha^{2} b^{\log _{a^{\prime}}\left(\theta_{2}\right)} f\left(\theta_{2}\right)\right]^{\log _{\theta_{2}}\left(\theta_{1}\right)}[\alpha f(1)]^{1 / \log _{\theta_{2}}\left(\theta_{1}\right)}, \\
& h\left(\theta_{1}\right)>h\left(\theta_{2}\right)^{\mu} h(1)^{1 / \mu} .
\end{aligned}
$$

On the other hand, applying the requisites over $a, a^{\prime}, b, b^{\prime}$ and $\alpha$ from Equation (2),

$$
\begin{aligned}
h\left(\theta_{1}\right) & =\alpha^{2} b^{\prime \log _{a^{\prime}}\left(\theta_{1}\right)} f\left(\theta_{1}\right)>\max \left\{\alpha^{2} b^{\prime \log _{a^{\prime}}\left(\theta_{2}\right)} f\left(\theta_{2}\right), \alpha f(1)\right\} \\
& =\max \left\{f\left(\theta_{2}\right), f(1)\right\},
\end{aligned}
$$

arriving at a contradiction.

Corollary 1. Let $f$ be an mc-function and $\theta_{1}<\theta_{2}<\theta_{3}$. Then, it cannot be $\max \left\{f\left(\theta_{1}\right), f\left(\theta_{3}\right)\right\}$ $<f\left(\theta_{2}\right)$. 
Proof. Assume otherwise. If $\theta_{1}=1$, then this corollary is just Theorem 6. For $1<\theta_{1}$, consider $g_{1}(x)=f\left(\theta_{1} x\right)$. For $\theta_{3}>1$, consider $g_{2}(x)=f\left(\frac{\theta_{3}}{x}\right)$.

For $\theta_{3}<1$, consider first $\tilde{g}_{3}(x)=f\left(\frac{1}{x}\right)$ and then $g_{3}(x)=\tilde{g}_{3}\left(\frac{1}{\theta_{3}} x\right)$.

In any case, $g_{i}$ would be an $m c$-function which contradicts Theorem 6 .

Corollary 2. Let $f$ be an mc-convex function which is not locally constant (that is, for every real number $x$ and interval I with $x$ in I we can find $x_{1} \neq x_{2}$ also in I so that $f\left(x_{1}\right) \neq f\left(x_{2}\right)$ ).

Then $f$ is at most 2-injective (meaning that for every real number $y \# f^{-1}(y) \leq 2$ ).

Proof. Let $y$ be a real number and $x_{1}, x_{2}, x_{3}$ be so that $f\left(x_{1}\right)=f\left(x_{2}\right)=f\left(x_{3}\right)$. Because $f$ is not locally constant, we can find $x_{1}<x^{(1)}<x_{2}<x^{(2)}<x_{3}$ so that $f\left(x^{(1)}\right) \neq f\left(x_{2}\right) \neq$ $f\left(x^{(2)}\right)$ and this is a contradiction with Theorem 6 .

The following Corollary generalizes Theorem 2.1 from [2]:

Corollary 3. Let $f$ be an mc-function (continuous or not). Then, $f$ is either monotone or decreasing-increasing.

Proof. This Corollary is easily proved if we consider the situation where $f$ is not monotone and we apply Theorem 6 repeatedly.

Corollary 3, in combination with Theorem 4 , implies that the set $\mathcal{M C}$ must be of cardinality less than $c$. On the other hand, since the function $f(x)=b$ is an $m c$-function for $b \geq 1$, we conclude the following Theorem, which answers several questions posted in [3]:

Theorem 7. The set $\mathcal{M C}$ has cardinality $\mathrm{c}$.

In particular, this implies that the algebraic structures considered in [3] are of the greatest possible dimension.

\section{On the Set of Points of Discontinuity of an $m c$-Function}

It is obvious that, if $A=\left\{x_{1}, x_{2}, \ldots, x_{n}\right\}$ is any finite set and, for $1 \leq k \leq n, f_{x_{k}}$ is an $m c$-function that is discontinuous at $x_{k}$, then $g(x)=f_{x_{1}}(x)+f_{x_{2}}(x)+\ldots+f_{x_{n}}(x)$ is discontinuous over the set $A$. The question we will focus on now is whether it is posible to find an $m c$-function which is discontinuous over an infinite set. We remark that, taking Theorem 4 and Corollary 3 into account, the set $A$ must be countable.

The main result of this section is as follows:

Theorem 8. Let $\mathcal{X}=\left\{x_{n}\right\}_{n=1}^{\infty} \subseteq(0,1)$ be a decreasing sequence. Then, there exists an mcfunction which is discontinuous on $\mathcal{X}$.

Before giving the proof, we will need some preliminary lemmas and definitions.

Lemma 2. Let $\Gamma$ be any index set, $\left\{f_{\alpha}\right\}_{\alpha \in \Gamma} \subseteq \mathcal{M C}$ and assume that, for $x>0$,

$$
\left\{f_{\alpha}(x): \alpha \in \Gamma\right\}
$$

is a bounded set.

Then,

$$
g(x)=\sup _{\alpha \in \Gamma}\left\{f_{\alpha}(x)\right\}
$$

is an mc-function. 
Proof. We shall use the following fact, if $\left\{y_{n}\right\}_{n=1}^{\infty}$ and $\left\{z_{n}\right\}_{n=1}^{\infty}$ are bounded sequences, then

$$
\lim _{n \rightarrow \infty} \sup _{k \geq n} y_{k} z_{k} \leq\left(\lim _{n \rightarrow \infty} \sup _{k \geq n} y_{k}\right)\left(\lim _{n \rightarrow \infty} \sup _{k \geq n} z_{k}\right) .
$$

Let now $y, z, \mu>0$. Then, we can find a sequence $\left\{\alpha_{n}\right\}_{n=1}^{\infty} \subseteq \Gamma$ so that $g\left(y^{\mu} z^{1 / \mu}\right)=$ $\lim _{n \rightarrow \infty} f_{\alpha_{n}}\left(y^{\mu} z^{1 / \mu}\right)$. Now,

$$
\begin{aligned}
g\left(y^{\mu} z^{1 / \mu}\right) & =\lim _{n \rightarrow \infty} f_{\alpha_{n}}\left(y^{\mu} z^{1 / \mu}\right) \\
& \leq \lim _{n \rightarrow \infty} \sup _{k \geq n} f_{\alpha_{k}}\left(y^{\mu} z^{1 / \mu}\right) \\
& \leq \lim _{n \rightarrow \infty} \sup _{k \geq n}\left[f_{\alpha_{k}}(y)^{\mu} f_{\alpha_{k}}(z)^{1 / \mu}\right] \\
& \leq\left[\lim _{n \rightarrow \infty} \sup _{k \geq n} f_{\alpha_{k}}(y)^{\mu}\right]\left[\lim _{n \rightarrow \infty} \sup _{k \geq n} f_{\alpha_{k}}(z)^{1 / \mu}\right] \\
& \leq g(y)^{\mu} g(z)^{1 / \mu} .
\end{aligned}
$$

Definition 4. Given a decreasing sequence $\left\{x_{n}\right\}_{n=1}^{\infty} \subset(0,1)$ and $n \geq 1$, we will define the following elements:

$$
\begin{aligned}
a_{n} & =\left(\frac{x_{n}}{x_{n+1}}\right)^{x_{n+1},}, \\
\alpha_{n} & =\prod_{k \geq n} a_{k}, \\
\beta_{n} & =\prod_{k \geq n} a_{k}^{2} .
\end{aligned}
$$

Lemma 3. For every $n \geq 1, \alpha_{n}$ and $\beta_{n}$ are well-defined.

Proof. We will just show that $\beta_{1}$ is well-defined.

Indeed, the product that defines $\beta_{1}$ converges if and only if $\sum_{k=1}^{\infty} \log \left(a_{k}^{2}\right)$ converges. Now,

$$
\begin{aligned}
\sum_{k=1}^{\infty} \log \left(a_{k}^{2}\right) & =2 \sum_{k=1}^{\infty} \log \left[\left(\frac{x_{k}}{x_{k+1}}\right)^{x_{k+1}}\right]=2 \sum_{k=1}^{\infty} x_{k+1} \log \left(\frac{x_{k}}{x_{k+1}}\right) \\
& =2 \sum_{k=1}^{\infty} x_{k+1} \log \left(1+\frac{x_{k}-x_{k+1}}{x_{k+1}}\right) \leq 2 \sum_{k=1}^{\infty} x_{k+1} \frac{x_{k}-x_{k+1}}{x_{k+1}} \\
& =2 \sum_{k=1}^{\infty}\left(x_{k}-x_{k+1}\right) \leq 4 x_{1} .
\end{aligned}
$$

Lemma 4. Let $n \geq 3,1 \leq m \leq n-2$ and $\left\{x_{k}\right\}_{k=1}^{\infty},\left\{a_{k}\right\}_{k=1}^{\infty}$ be as in Definition 4. Then,

$$
\left(\frac{x_{n}}{x_{m}}\right)^{x_{m}} \prod_{k=m}^{n-1} a_{k}<\left(\frac{x_{n}}{x_{m+1}}\right)^{x_{m+1}} \prod_{k=m+1}^{n-1} a_{k}
$$


Proof. Just notice that

$$
\begin{aligned}
\left(\frac{x_{n}}{x_{m}}\right)^{x_{m}} \prod_{k=m}^{n-1} a_{k} & =\left(\frac{x_{n}}{x_{m}}\right)^{x_{m}} a_{m} \prod_{k=m+1}^{n-1} a_{k} \\
& =\left(\frac{x_{n}}{x_{m}}\right)^{x_{m}}\left(\frac{x_{m}}{x_{m+1}}\right)^{x_{m+1}} \prod_{k=m+1}^{n-1} a_{k} \\
& =\left(\frac{x_{n}}{x_{m}}\right)^{x_{m}}\left(\frac{x_{m}}{x_{m+1}}\right)^{x_{m+1}}\left(\frac{x_{m+1}}{x_{n}}\right)^{x_{m+1}}\left(\frac{x_{n}}{x_{m+1}}\right)^{x_{m+1}} \prod_{k=m+1}^{n-1} a_{k} \\
& =\left(\frac{x_{n}}{x_{m}}\right)^{x_{m}-x_{m+1}}\left(\frac{x_{n}}{x_{m+1}}\right)^{x_{m+1}} \prod_{k=m+1}^{n-1} a_{k} \\
& <\left(\frac{x_{n}}{x_{m+1}}\right)^{x_{m+1}} \prod_{k=m+1}^{n-1} a_{k}
\end{aligned}
$$

based on the fact that $\left\{x_{k}\right\}_{k=1}^{\infty}$ is a decreasing sequence.

Lemma 5. Let $\left\{x_{n}\right\}_{n=1}^{\infty} \subset(0,1)$ be a decreasing sequence. Then, for every $n \geq 1$ and $m \geq n+2$,

$$
\left(\frac{x_{n}}{x_{n+1}}\right)^{x_{n+1}}\left(\frac{x_{n+1}}{x_{n+2}}\right)^{x_{n+2}} \cdot \ldots \cdot\left(\frac{x_{m-1}}{x_{m}}\right)^{x_{m}}>\left(\frac{x_{n}}{x_{m}}\right)^{x_{m}}
$$

Proof. We will proceed by induction on $m$. If $m=n+2$, then

$$
\begin{aligned}
\left(\frac{x_{n}}{x_{n+1}}\right)^{x_{n+1}}\left(\frac{x_{n+1}}{x_{n+2}}\right)^{x_{n+2}} & =\left(\frac{x_{n}}{x_{n+1}}\right)^{x_{n+1}-x_{n+2}}\left(\frac{x_{n}}{x_{n+1}}\right)^{x_{n+2}}\left(\frac{x_{n+1}}{x_{n+2}}\right)^{x_{n+2}} \\
& =\left(\frac{x_{n}}{x_{n+1}}\right)^{x_{n+1}-x_{n+2}}\left(\frac{x_{n}}{x_{n+2}}\right)^{x_{n+2}} \\
& >\left(\frac{x_{n}}{x_{n+2}}\right)^{x_{n+2}} .
\end{aligned}
$$

Assuming the result is true for $m$, then

$$
\begin{aligned}
\left(\frac{x_{n}}{x_{n+1}}\right)^{x_{n+1}} & \left(\frac{x_{n+1}}{x_{n+2}}\right)^{x_{n+2}} \cdot \ldots \cdot\left(\frac{x_{m-1}}{x_{m}}\right)^{x_{m}}\left(\frac{x_{m}}{x_{m+1}}\right)^{x_{m+1}} \\
& >\left(\frac{x_{n}}{x_{m}}\right)^{x_{m}}\left(\frac{x_{m}}{x_{m+1}}\right)^{x_{m+1}} \\
& =\left(\frac{x_{n}}{x_{m}}\right)^{x_{m}-x_{m+1}}\left(\frac{x_{n}}{x_{m}}\right)^{x_{m+1}}\left(\frac{x_{m}}{x_{m+1}}\right)^{x_{m+1}} \\
& >\left(\frac{x_{n}}{x_{m+1}}\right)^{x_{m+1}}
\end{aligned}
$$

as desired.

Corollary 4. For every $n \geq 1$ and $m \geq n+2$,

$$
\begin{aligned}
& \alpha_{m}\left(\frac{x_{n}}{x_{m}}\right)^{x_{m}}<\alpha_{n+1}\left(\frac{x_{n}}{x_{n+1}}\right)^{x_{n+1}} \text { and } \\
& \beta_{m}\left(\frac{x_{n}}{x_{m}}\right)^{x_{m}}<\beta_{n+1}\left(\frac{x_{n}}{x_{n+1}}\right)^{x_{n+1}},
\end{aligned}
$$

where $x_{k}, \alpha_{k}$ and $\beta_{k}$ are as in Definition 4. 
Theorem 9. Let $y_{n} \rightarrow y_{0}$ and $\left\{f_{k}\right\}_{k=1}^{\infty} \subseteq \mathbb{R}^{\mathbb{R}}$. Then,

$$
\sup _{k \in \mathbb{N}} \lim _{n \rightarrow \infty}\left\{\sup _{m \geq n} f_{k}\left(y_{m}\right)\right\} \leq \lim _{n \rightarrow \infty} \sup _{m \geq n}\left\{\sup _{k \in \mathbb{N}} f_{k}\left(y_{m}\right)\right\} .
$$

Proof. Let $l, n \in \mathbb{N}$. We notice that

$$
f_{l}\left(y_{n}\right) \leq \sup _{k \in \mathbb{N}} f_{k}\left(y_{n}\right)
$$

Therefore,

$$
\sup _{m \geq n} f_{l}\left(y_{m}\right) \leq \sup _{m \geq n}\left\{\sup _{k \in \mathbb{N}} f_{k}\left(y_{m}\right)\right\}
$$

and hence

$$
\lim _{n \rightarrow \infty}\left\{\sup _{m \geq n} f_{l}\left(y_{m}\right)\right\} \leq \lim _{n \rightarrow \infty} \sup _{m \geq n}\left\{\sup _{k \in \mathbb{N}} f_{k}\left(y_{m}\right)\right\}
$$

for every $l \in \mathbb{N}$. Finally,

$$
\sup _{k \in \mathbb{N}} \lim _{n \rightarrow \infty}\left\{\sup _{m \geq n} f_{k}\left(y_{m}\right)\right\} \leq \lim _{n \rightarrow \infty} \sup _{m \geq n}\left\{\sup _{k \in \mathbb{N}} f_{k}\left(y_{m}\right)\right\}
$$

Corollary 5. Let $\left\{f_{k}\right\}_{k=1}^{\infty}$ be a sequence of functions so that, for every $x \in \mathbb{R},\left\{f_{k}(x): k \geq 1\right\}$ is a bounded set and $\lim _{x \rightarrow x_{0}} f_{k}(x)$ exists for every $k \geq 1$. Define the function $g(x)=\sup _{k \geq 1} f_{k}(x)$. Then, if $\lim _{x \rightarrow x_{0}} g(x)$ also exists,

$$
\sup _{k \geq 1} \lim _{x \rightarrow x_{0}} f_{k}(x) \leq \lim _{x \rightarrow x_{0}} g(x)=\lim _{x \rightarrow x_{0}} \sup _{k \geq 1} f_{k}(x)
$$

Remark 1. Equality in (3) cannot generally be attained: as a counterexample, we choose the functions

$$
f_{k}(x)=1-\left(\frac{1}{1+x}\right)^{k}, x>-1, k \geq 0 .
$$

Then, $\lim _{x \rightarrow 0} f_{k}(x)=0$ for every $k \geq 1$, so that $\sup _{k \geq 1} \lim _{x \rightarrow 0} f_{k}(x)$. On the other hand, $\sup _{k \geq 1} f_{k}(x)=1$ for every $k \geq 1, x>-1$, so that $\lim _{x \rightarrow 0} \sup _{k \geq 1} f_{k}(x)=1$.

Proof of Theorem 8. Define, given $m \in \mathbb{N}$, the following function:

$$
f_{m}(t)= \begin{cases}\alpha_{m}\left(\frac{t}{x_{m}}\right)^{x_{m}} & \text { if } 0<t \leq x_{m} \\ \beta_{m}\left(\frac{t}{x_{m}}\right)^{x_{m}} & \text { if } t>x_{m}\end{cases}
$$

where $x_{m}, \alpha_{m}$ and $\beta_{m}$ are as in Definition 4 .

The function $f_{m}$ is multiplicative convex and it is discontinuous at $t=x_{m}$, since it has been defined following the contruction from Proposition 1.

We will show that the function $g(t)=\sup \left\{f_{n}(t): n \in \mathbb{N}\right\}$ is discontinuous on the set $\mathcal{X}=\left\{x_{n}\right\}_{n=1}^{\infty}$. First of all, for $t>0$ the set $\left\{f_{n}(t): n \in \mathbb{N}\right\}$ is bounded since

$$
\begin{aligned}
\alpha_{n}, \beta_{n} & \leq \beta_{1} \leq e^{4 x_{1}}, \\
t^{x_{n}} & \leq\left\{\begin{array}{ll}
1 & \text { if } 0<t \leq 1, \\
t & \text { if } t>1,
\end{array} \text { for every } n \geq 1\right. \text { and } \\
x_{n}^{-x_{n}} & \leq e^{1 / e}
\end{aligned}
$$

because of Lemma 1. 
Let $n, m \in \mathbb{N}$. Then,

$$
f_{m}\left(x_{n}\right)= \begin{cases}\alpha_{m}\left(\frac{x_{n}}{x_{m}}\right)^{x_{m}} & \text { if } 1 \leq m \leq n \\ \beta_{m}\left(\frac{x_{n}}{x_{m}}\right)^{x_{m}} & \text { if } m \geq n+1\end{cases}
$$

Notice that, if $m<n$

$$
\begin{aligned}
\alpha_{m}\left(\frac{x_{n}}{x_{m}}\right)^{x_{m}}= & \left(\frac{x_{n}}{x_{m}}\right)^{x_{m}} \prod_{k \geq m} a_{k} \\
= & \left(\frac{x_{n}}{x_{m}}\right)^{x_{m}}\left(\frac{x_{m}}{x_{m+1}}\right)^{x_{m+1}}\left(\frac{x_{m+1}}{x_{m+2}}\right)^{x_{m+2}} \cdot \ldots \\
& \ldots \cdot\left(\frac{x_{n-1}}{x_{n}}\right)^{x_{n}} \prod_{k \geq n}\left(\frac{x_{k}}{x_{k+1}}\right)^{x_{k+1}} \\
= & \left(\frac{x_{n}}{x_{m}}\right)^{x_{m}}\left(\frac{x_{m}}{x_{m+1}}\right)^{x_{m+1}}\left(\frac{x_{m+1}}{x_{m+2}}\right)^{x_{m+2}} \ldots \cdot\left(\frac{x_{n-1}}{x_{n}}\right)^{x_{n}} \alpha_{n} \\
< & \left(\frac{x_{n}}{x_{n-1}}\right)^{x_{n-1}}\left(\frac{x_{n-1}}{x_{n}}\right)^{x_{n}} \alpha_{n} \\
< & \alpha_{n}
\end{aligned}
$$

by Lemma 4 .

If $m \geq n+2$, and using Corollary 4 , we can prove that

$$
\begin{aligned}
\beta_{n} & =\left(\frac{x_{n}}{x_{n+1}}\right)^{2 x_{n+1}} \beta_{n+1} \\
& >\left(\frac{x_{n}}{x_{n+1}}\right)^{x_{n+1}} \beta_{n+1} \\
& >\left(\frac{x_{n}}{x_{m}}\right)^{x_{m}} \beta_{m} .
\end{aligned}
$$

In conclusion, we can say that $g\left(x_{n}\right)<\beta_{n}$.

On the other hand,

$$
\lim _{x \rightarrow x_{n}^{+}} f_{m}(x)= \begin{cases}\alpha_{m}\left(\frac{x_{n}}{x_{m}}\right)^{x_{m}} & \text { if } 1 \leq m \leq n-1, \\ \beta_{m}\left(\frac{x_{n}}{x_{m}}\right)^{x_{m}} & \text { if } m \geq n\end{cases}
$$

With a similar argument as before, $\sup \left\{\lim _{x \rightarrow x_{n}^{+}} f_{m}(x): m \geq 1\right\}=\beta_{n}$.

Hence, by Corollary 5

$$
\begin{aligned}
\lim _{x \rightarrow x_{n}^{+}} g(x) & =\lim _{x \rightarrow x_{n}^{+}} \sup \left\{f_{m}(x): m \geq 1\right\} \\
& \geq \sup \left\{\lim _{x \rightarrow x_{n}^{+}} f_{m}(x): m \geq 1\right\} \\
& =\beta_{n}>g\left(x_{n}\right) .
\end{aligned}
$$

As a consequence, $g$ is not continuous at $x_{n}$.

The following result complements Theorem 8 and shows that $\mathfrak{D}(g)=\mathcal{X}$ :

Proposition 3. The function $g$ considered in the proof of Theorem 8 is continuous on $(0, \infty) \backslash \mathcal{X}$. 
Proof. Let $f_{m}$ be the functions defined in the proof of Theorem $8, m_{0} \geq 1$ and $x_{m_{0}}<x<$ $x_{m_{0}-1}$. Then,

$$
f_{m}(x)= \begin{cases}\alpha_{m}\left(\frac{x}{x_{m}}\right)^{x_{m}} & \text { if } m \leq m_{0}-1, \\ \beta_{m}\left(\frac{x}{x_{m}}\right)^{x_{m}} & \text { if } m \geq m_{0} .\end{cases}
$$

If $m \leq m_{0}-1$, then $x<x_{m}$ so that

$$
\begin{aligned}
& x_{m}^{x_{m+1}-x_{m}} \leq x^{x_{m+1}-x_{m}}, \quad \text { from which } \\
& \left(\frac{x_{m}}{x_{m+1}}\right)^{x_{m+1}}\left(\frac{x}{x_{m}}\right)^{x_{m}} \leq\left(\frac{x}{x_{m+1}}\right)^{x_{m+1}} \text { and therefore } \\
& \alpha_{m}\left(\frac{x}{x_{m}}\right)^{x_{m}} \leq \alpha_{m+1}\left(\frac{x}{x_{m+1}}\right)^{x_{m+1}}
\end{aligned}
$$

leading us to conclude that

$$
\sup \left\{f_{m}(x): 1 \leq m \leq m_{0}-1\right\}=\alpha_{m_{0}-1}\left(\frac{x}{x_{m_{0}-1}}\right)^{x_{m_{0}-1}} .
$$

If now $m \geq m_{0}$, then $x_{m}<x$, which implies that $x^{x_{m+1}-x_{m}}<x_{m}^{x_{m+1}-x_{m}}$. On the other hand, from $x_{m+1}<x_{m}$ we can deduce that

$$
x_{m}^{x_{m+1}-x_{m}}<\frac{x_{m}^{2 x_{m+1}-x_{m}}}{x_{m+1}^{x_{m+1}}},
$$

which allows us to deduce that

$$
x^{x_{m+1}-x_{m}}<\frac{x_{m}^{2 x_{m+1}-x_{m}}}{x_{m+1}^{x_{m+1}}} .
$$

This last inequality is equivalent to

$$
\left(\frac{x}{x_{m+1}}\right)^{x_{m+1}}<\left(\frac{x_{m}}{x_{m+1}}\right)^{2 x_{m+1}}\left(\frac{x}{x_{m}}\right)^{x_{m}},
$$

that is,

$$
\beta_{m+1}\left(\frac{x}{x_{m+1}}\right)^{x_{m+1}}<\beta_{m}\left(\frac{x}{x_{m}}\right)^{x_{m}}
$$

As a consequence,

$$
\sup \left\{f_{m}(x): m \geq m_{0}\right\}=\beta_{m_{0}}\left(\frac{x}{x_{m_{0}}}\right)^{x_{m_{0}}}
$$

and therefore

$$
\begin{aligned}
g(x) & =\sup \left\{f_{m}(x): m \geq 1\right\} \\
& =\max \left\{\alpha_{m_{0}-1}\left(\frac{x}{x_{m_{0}-1}}\right)^{x_{m_{0}-1}}, \beta_{m_{0}}\left(\frac{x}{x_{m_{0}}}\right)^{x_{m_{0}}}\right\} .
\end{aligned}
$$

Hence, on $\left(x_{m} \cdot x_{m-1}\right), g$ can be expressed as the maximum of two continuous functions and in conclusion it is continuous over that interval.

If next $0<x<x_{0}=\lim _{n \rightarrow \infty} x_{n}$ (which in particular implies that $x_{0} \neq 0$ ), then $f_{m}(x)=\alpha_{m}\left(\frac{x}{x_{m}}\right)^{x_{m}}$ and, as above, we would have

$$
\alpha_{m}\left(\frac{x}{x_{m}}\right)^{x_{m}} \leq \alpha_{m+1}\left(\frac{x}{x_{m+1}}\right)^{x_{m+1}}
$$


Therefore,

$$
g(x)=\lim _{m \rightarrow \infty} \alpha_{m}\left(\frac{x}{x_{m}}\right)^{x_{m}}=\left(\frac{x}{x_{0}}\right)^{x_{0}},
$$

which again is a continuous function.

For the case $x=x_{0}$ we notice that $g\left(x_{0}\right)=1$. Assume $\left\{y_{n}\right\}_{n=1}^{\infty}$ is such that $y_{n} \rightarrow x_{0}$ as $n \rightarrow \infty$. If $y_{n}<x_{0}$ then $g\left(y_{n}\right)=\left(\frac{y_{n}}{x_{0}}\right)^{x_{0}}$. On the other hand, if $y_{n}>x_{0}$ then we can find $\left\{m_{n}\right\}_{n=1}^{\infty} \subseteq \mathbb{N}$ so that $x_{m_{n}} \leq y_{n}<x_{m_{n}-1}$. As before,

$$
g\left(y_{n}\right)=\max \left\{\alpha_{m_{n}-1}\left(\frac{y_{n}}{x_{m_{n}-1}}\right)^{x_{m_{n}-1}}, \beta_{m_{n}}\left(\frac{y_{n}}{x_{m_{n}}}\right)^{x_{m_{n}}}\right\} \text {. }
$$

Since

$$
\lim _{n \rightarrow \infty}\left(\frac{y_{n}}{x_{0}}\right)^{x_{m_{0}}}=\lim _{n \rightarrow \infty} \alpha_{m_{n}-1}\left(\frac{y_{n}}{x_{m_{n}-1}}\right)^{x_{m_{n}-1}}=\lim _{n \rightarrow \infty} \beta_{m_{n}}\left(\frac{y_{n}}{x_{m_{n}}}\right)^{x_{m_{n}}}=1,
$$

we are able to conclude that $\lim _{n \rightarrow \infty} g\left(y_{n}\right)=g\left(x_{0}\right)$.

Finally, for the case $x>x_{1}$ we would obtain that $f_{m}(x)=\beta_{m}\left(\frac{x}{x_{m}}\right)^{x_{m}}$ and hence (again, similarly as before)

$$
g(x)=\beta_{1}\left(\frac{x}{x_{1}}\right)^{x_{1}}
$$

so $g$ is continuous on $x$.

Remark 2. If $\mathcal{X} \subseteq(0,1)$ is an increasing sequence, then there exists an mc-function so that $\mathfrak{D}(f)=\mathcal{X}$.

Indeed, we only need to change the definition of the elements $a_{n}$ in Definition 4 as follows:

$$
a_{n}=\left(\frac{x_{n+1}}{x_{n}}\right)^{x_{n}}
$$

Corollary 6. If $\mathcal{X}$ is a monotone sequence, then we can find an mc-function which is discontinuous on $\mathcal{X}$.

Proof. Let $\mathcal{X}_{1}=\mathcal{X} \cap(0,1)$ and $\mathcal{X}_{2}=\mathcal{X} \cap[1, \infty)$. We can find two functions $f_{1}$ and $f_{2}$ so that $f_{1}$ is discontinuous only on $\mathcal{X}_{1}$ and $f_{2}$ is discontinuous only on the set $\left\{\frac{1}{x}: x \in \mathcal{X}_{2}\right\}$.

The required function is then $f(x)=f_{1}(x)+f_{2}\left(\frac{1}{x}\right)$.

\section{Algebraic Structure on $\mathcal{M C} \backslash C(0, \infty)$}

Theorem 10. There exists an algebraic truncated cone of algebraic dimension $\mathfrak{c}$ every non-trivial algebraic combination of which is an mc-function which is discontinuous at infinitely many points.

Proof. Let us consider a $\mathbb{Q}$-linearly independent set of cardinality $\mathfrak{c},\left\{a_{\zeta}: \zeta<\mathfrak{c}\right\} \subseteq(4,6)$. Then, for every $\zeta<\mathfrak{c}$ we consider a decreasing sequence, $\mathcal{X}_{\alpha}=\left\{x_{k, \zeta}\right\}_{k=1}^{\infty}$ converging to $\frac{1}{a_{\zeta}}$ and so that $x_{1, \zeta}=\frac{1}{4}$.

Using Theorem 8 for every $\zeta<\mathfrak{c}$ there exists an $m c$-function $\tilde{f}_{\zeta}$ which is discontinuous on $\mathcal{X}_{\zeta}$.

In particular, taking a look at the proof of Theorem 8 , the function $\tilde{f}_{\zeta}$ is defined as $\tilde{f}_{\zeta}(x)=\sup \left\{f_{k, \zeta}(x): k \in \mathbb{N}\right\}$, where 


$$
\begin{aligned}
f_{k, \zeta}(x) & =\left\{\begin{array}{ll}
\alpha_{k, \zeta}\left(\frac{x}{x_{k, \zeta}}\right)^{x_{k, \zeta}} & \text { if } 0<x \leq x_{k, \zeta}, \\
\beta_{k, \zeta}\left(\frac{x}{x_{k, \zeta}}\right)^{x_{k, \zeta}} & \text { if } x>x_{k, \zeta}
\end{array},\right. \\
\alpha_{k, \zeta} & =\prod_{l \geq n}\left(\frac{x_{l, \zeta}}{x_{l+1, \zeta}}\right), \\
\beta_{k, \zeta} & =\prod_{l \geq n}\left(\frac{x_{l, \zeta}}{x_{l+1, \zeta}}\right)^{2} .
\end{aligned}
$$

In particular, if $x>x_{1, \zeta}$ then

$$
\tilde{f}_{\zeta}(x)=\sup \left\{\beta_{k, \zeta}\left(\frac{x}{x_{k, \zeta}}\right)^{x_{k, \zeta}}: k \in \mathbb{N}\right\} .
$$

Because of Lemma 1 and since $\left\{\frac{1}{x_{k, \zeta}}\right\}_{k=1}^{\infty}$ is an increasing sequence with $\frac{1}{x_{1, \zeta}}=4>e$, we obtain that if $x>x_{1, \zeta}=\frac{1}{4}$ then $\tilde{f}_{\zeta}(x)=\beta_{1, \zeta}(4 x)^{1 / 4}$.

Define, for $\zeta<\mathfrak{c}$, the auxiliary function

$$
g_{\zeta}(x)=\frac{4}{4^{1 / 4} \beta_{1, \zeta}} x^{a_{\zeta}-1 / 4}
$$

From the proof of Lemma $3, \beta_{1, \zeta}<e^{4 x_{1, \zeta}}=e$ and therefore

$$
1<\frac{4}{4^{1 / 4} \beta_{1, \zeta}}
$$

so $g_{\zeta}$ is a (continuous) $m c$-function.

Define then the function

$$
f_{\zeta}(x)=\tilde{f}_{\zeta}(x) g_{\zeta}(x) .
$$

Then $f_{\zeta}$ is an $m c$-function (because it is the product of two $m c$-functions) which is discontinuous on the set $\mathcal{X}_{\zeta}$.

Furthermore, if $\frac{1}{4}<x$,

$$
f_{\zeta}(x)=\tilde{f}_{\zeta}(x) g_{\zeta}(x)=\beta_{1, \zeta}(4 x)^{1 / 4} \frac{4}{4^{1 / 4} \beta_{1, \zeta}} x^{a_{\zeta}-1 / 4}=4 x^{a_{\zeta}} .
$$

Let us show that $B=\left\{f_{\zeta}: \zeta<\mathfrak{c}\right\}$ is an algebraically independent set.

Indeed, let $\zeta_{1}, \zeta_{2}, \ldots, \zeta_{n}<\mathfrak{c}, \lambda_{1}, \ldots, \lambda_{m}$ be non-zero numbers and $N=\left(n_{i, j}\right)_{i, j=1}^{n, m}$ be a matrix consisting of natural numbers as entries and without two equal columns.

Assume that

$$
f=\sum_{j=1}^{m} \lambda_{j} \prod_{i=1}^{n} f_{\zeta_{i}}^{n_{i, j}}=0
$$

Then, we notice that for $\frac{1}{4}<x$ it must be

$$
\begin{aligned}
0 & =f(x)=\sum_{j=1}^{m} \lambda_{j} \prod_{i=1}^{n} f_{\zeta_{i}}^{n_{i, j}}(x) \\
& =\sum_{j=1}^{m} \lambda_{j} \prod_{i=1}^{n}\left(4 x^{a_{\zeta_{i}}}\right)^{n_{i, j}} \\
& =\sum_{j=1}^{m} \lambda_{j}\left(4^{\sum_{i=1}^{n} n_{i, j}}\right) x^{\sum_{i=1}^{n} a_{\zeta_{i}} n_{i, j}} .
\end{aligned}
$$


Since the elements $a_{\zeta_{1}}, a_{\zeta_{2}}, \ldots, a_{\zeta_{n}}$ are $\mathbb{Q}$-linearly independent and the columns of $N=\left\{n_{i, j}\right\}_{i, j=1}^{n, m}$ are pairwise different, we can conclude that the exponents

$$
\sum_{i=1}^{n} a_{\zeta_{i}} n_{i, j}
$$

are all different from each other.

Therefore, $f_{\mid(1 / 4, \infty)}$ is an identically null extended polynomial (with positive exponents). Hence, all its coefficients must be zero and, in conclusion, if $1 \leq j \leq m$

$$
\lambda_{j} 4^{\sum_{i=1}^{n} n_{i, j}}=0 \text {, which implies that } \lambda_{j}=0
$$

and in conclusion $B$ is algebraically independent.

To finish with, let us choose an element $f$ in the truncated cone generated by $B$. Then, we can find $\zeta_{1}, \zeta_{2}, \ldots, \zeta_{n}<\mathfrak{c}, \lambda_{1}, \ldots, \lambda_{m} \geq 1$ and a matrix consisting of natural numbers as entries and without two equal columns $N=\left\{n_{i, j}\right\}_{i, j=1}^{n, m}$ so that

$$
f=\sum_{j=1}^{m} \lambda_{j} \prod_{i=1}^{n} f_{\zeta_{i}}^{n_{i, j}} .
$$

Without loss of generality we may assume that $a_{\zeta_{i}}<a_{\zeta_{i+1}}$ for every $1 \leq i \leq n-1$. Then, $f_{\zeta_{i}}$ is continuous on $\left(0, a_{\zeta_{2}}\right)$ for every $i \geq 2$.

For any function $h: \mathbb{R} \rightarrow \mathbb{R}$ we denote $h^{a^{-}}=\lim _{x \rightarrow a^{-}} h(x)$ and $h^{a^{+}}=\lim _{x \rightarrow a^{+}} h(x)$. We can then write

$$
f=f_{\zeta_{1}}^{n_{1,1}} g_{1}+\ldots+f_{\zeta_{1}}^{n_{j, 1}} g_{j}+\ldots+f_{\zeta_{1}}^{n_{m}, 1} g_{m}
$$

where $g_{j}$ is an $m c$-function continuous on $\left(0, a_{\zeta_{2}}\right)$

For every $\beta \in \mathcal{X}_{\zeta_{i}} \cap\left(0, a_{\zeta_{2}}\right)$, we have that

$$
f^{\beta^{+}}=\left(f_{\zeta_{1}}^{\beta^{+}}\right)^{n_{1,1}} g_{1}(\beta)+\ldots+\left(f_{\zeta_{1}}^{\beta^{+}}\right)^{n_{j, 1}} g_{j}(\beta)+\ldots+\left(f_{\zeta_{1}}^{\beta^{+}}\right)^{n_{m, 1}} g_{1}(\beta)
$$

and

$$
f^{\beta^{-}}=\left(f_{\zeta_{1}}^{\beta^{-}}\right)^{n_{1,1}} g_{1}(\beta)+\ldots+\left(f_{\zeta_{1}}^{\beta^{-}}\right)^{n_{j, 1}} g_{j}(\beta)+\ldots+\left(f_{\zeta_{1}}^{\beta^{-}}\right)^{n_{m, 1}} g_{m}(\beta) .
$$

Since the function $f_{\zeta_{1}}$ is not continuous at $\beta$ we may assume without loss of generality that $f_{\zeta_{1}}^{\beta^{-}}<f_{\zeta_{1}}^{\beta^{+}}$(the procedure would be analogous for the case $f_{\zeta_{1}}^{\beta^{-}}>f_{\zeta_{1}}^{\beta^{+}}$) and observe that if $f_{\zeta_{1}}^{\beta^{-}}<f_{\zeta_{1}}^{\beta^{+}}$then $\left(f_{\zeta_{1}}^{\beta^{-}}\right)^{q}<\left(f_{\zeta_{1}}^{\beta^{+}}\right)^{q}$ for every $q \in \mathbb{N} \backslash\{0\}$. We can now write

$$
\begin{aligned}
f^{\beta^{+}}-f^{\beta^{-}}= & \left(f_{\zeta_{1}}^{\beta^{+}}\right)^{n_{1,1}} g_{1}(\beta)+\ldots+\left(f_{\zeta_{1}}^{\beta^{+}}\right)^{n_{m, 1}} g_{m}(\beta) \\
& -\left[\left(f_{\zeta_{1}}^{\beta^{-}}\right)^{n_{1,1}} g_{1}(\beta)+\ldots+\left(f_{\zeta_{1}}^{\beta^{-}}\right)^{n_{m, 1}} g_{m}(\beta)\right] \\
= & g_{1}(\beta)\left[\left(f_{\zeta_{1}}^{\beta^{+}}\right)^{n_{1,1}}-\left(f_{\zeta_{1}}^{\beta^{-}}\right)^{n_{1,1}}\right]+\ldots+g_{j}(\beta)\left[\left(f_{\zeta_{1}}^{\beta^{+}}\right)^{n_{j, 1}}-\left(f_{\zeta_{1}}^{\beta^{-}}\right)^{n_{j, 1}}\right] \\
& +\ldots+g_{m}(\beta)\left[\left(f_{\zeta_{1}}^{\beta^{+}}\right)^{n_{m, 1}}-\left(f_{\zeta_{1}}^{\beta^{-}}\right)^{n_{m, 1}}\right] \\
> & 0,
\end{aligned}
$$

so that $f$ is discontinuous on $\mathcal{X}_{\zeta_{1}} \cap\left(0, a_{\zeta_{2}}\right)$ and the proof is done.

\section{Conclusions}

In [2] the authors focused on those $m c$-functions that attained the value 1 at $x=1$. It was proved that such functions were continuous and that in fact they could only be monotone, or decreasing until $x=1$ and increasing from $x=1$. In [3] some discontinuous 
$m c$-functions were introduced. This paper completes both of them in setting the cardinality of the set of all $m c$-functions at $c$ (and therefore "there are not really many more $m c$-functions out of the cathegory of continuous functions") and showing that every of these functions show either monotonous or decreasing-increasing behavior.

It also continues the ideas shown in [3] when it constructs a trunkated cone consisting on $m c$-functions which this time have infinitely many points of discontinuity.

There are still some questions that remain open, namely whether the set of discontinuous $m c$-functions is a truncated cone itself, and what kind of properties can be concluded for the set of points of discontinuity of an $m c$-function.

Author Contributions: Investigation, P.J.-R., M.E.M.-G., G.A.M.-F. and J.B.S.-S. All authors contributed equally to this work. All authors have read and agreed to the published version of the manuscript.

Funding: Partially supported by grant PGC2018-097286-B-I00.

Institutional Review Board Statement: Not applicable.

Informed Consent Statement: Not applicable.

Data Availability Statement: Not applicable.

Conflicts of Interest: The authors declare no conflict of interest.

\section{References}

1. Niculescu, C.P. Convexity according to the geometric mean. Math. Inequal. Appl. 2000, 2, 155-167. [CrossRef]

2. Jiménez-Rodríguez, P.; Martínez-Gómez, M.E.; Muñoz-Fernández, G.A.; Seoane-Sepúlveda, J.B. Describing multiplicative convex functions. J. Convex Anal. 2020, 27, 935-942.

3. Jiménez-Rodríguez, P.; Martínez-Gómez, M.E.; Muñoz-Fernández, G.A.; Seoane-Sepúlveda, J.B. Generalizing multiplicative convex functions. J. Convex Anal. 2021, 28.

4. Aron, R.M.; Gurariy, V.I.; Seoane Sepúlveda, J.B. Lineability and spaceability of sets of functions on R. Proc. Am. Math. Soc. 2005, 133, 795-803. [CrossRef]

5. Aron, R.M.; Bernal, González, L.; Pellegrino, D.M.; Seoane Sepúlveda, J.B. Lineability: The Search for Linearity in Mathematics; Monographs and Research Notes in Mathematics; CRC Press: Boca Raton, FL, USA, 2016; pp. xix+308, ISBN 978-1-4822-9909-0.

6. Bastin, F.; Conejero, J.A.; Esser, C.; Seoane-Sepúlveda, J.B. Algebrability and nowhere Gevrey differentiability. Israel J. Math. 2015, 205, 127-143. [CrossRef]

7. Bernal-González, L.; Pellegrino, D.; Seoane-Sepúlveda, J.B. Linear subsets of nonlinear sets in topological vector spaces. Bull. Am. Math. Soc. 2014, 51, 71-130. [CrossRef]

8. Ciesielski, K.; Seoane-Sepúlveda, J. Differentiability versus continuity: Restriction and extension theorems and monstrous examples. Bull. Am. Math. Soc. 2019, 56, 211-260. [CrossRef]

9. Froda, A. Sur la Distribution des Propriétés de Voisinage des Fonctions de Variables Réelles. Ph.D. Thesis, Paris Science Faculty, Paris France, 1929.

10. Moschovakis, Y. Notes on set theory. In Undergraduate Texts in Mathematics, 2nd ed.; Springer: New York, NY, USA, 2006; p. xii+276, ISBN 978-0387-28722-5.

11. Steiner, J. Über das größte Product der Theile oder Summanden jeder Zahl. Journal für die Reine und Angewandte Mathematik 1850, 40, 208-208. 